\title{
Mitochondrial Respiration of Human Platelets in Young Adult and Advanced Age - Seahorse or O2k?
}

\author{
Jan JEDLIČKA ${ }^{1,2^{*}}$, Radovan KUNC ${ }^{3,4^{*}}$, Jitka KUNCOVÁ ${ }^{1,2}$ \\ * These authors contributed equally to this work. \\ ${ }^{1}$ Institute of Physiology, Faculty of Medicine in Plzeň, Charles University, Plzeň, Czech Republic, \\ ${ }^{2}$ Biomedical Centre, Faculty of Medicine in Plzeň, Charles University, Plzeň, Czech Republic, \\ ${ }^{3}$ Institute of Social Medicine, Faculty of Medicine in Plzeň, Charles University, Plzeň, Czech \\ Republic, ${ }^{4}$ Hospice of St. Lazar, Plzeň, Czech Republic
}

Received April 19, 2021

Accepted October 12, 2021

\section{Summary}

The objective of the present study was to evaluate platelet mitochondrial oxygen consumption using high-resolution respirometry (HRR) and metabolic flux analysis (MFA) and to verify the effect of advanced age on these parameters. HRR was used to analyze permeabilized and intact platelets, MFA to measure oxygen consumption rates (OCR), extracellular acidification rates (ECAR) and ATP production rate in intact fixed platelets. Two groups of healthy volunteers were included in the study: YOUNG (20-42 years, $\mathrm{n}=44$ ) and older adults (OLD; 70-89 years; $n=15$ ). Compared to YOUNG donors, platelets from group OLD participants displayed significantly lower values of oxygen consumption in the Complex II-linked phosphorylating and uncoupled states and the Complex IV activity in HRR protocols for permeabilized cells and significantly lower resting and uncoupled respirations in intact cells when analyzed by both methods. In addition, mitochondrial ATP production rate was also significantly lower in platelets isolated from older adults. Variables measured by both methods from the same bloods correlated significantly, nevertheless those acquired by MFA were higher than those measured using HRR. In conclusion, the study verifies compromised mitochondrial respiration and oxidative ATP production in the platelets of aged persons and documents good compatibility of the two most widely used methods for determining the global performance of the electron-transporting system, i.e. HRR and MFA.

\section{Key words}

Platelets • Mitochondrial respiration • Aging • High resolution respirometry • Metabolic flux analysis

\section{Corresponding author}

J. Kuncová, Institute of Physiology, Faculty of Medicine in Plzeň, Charles University, Alej Svobody 76, 323 00, Plzeň, Czech Republic. E-mail: jitka.kuncova@lfp.cuni.cz

\section{Introduction}

Mitochondrial dysfunction is associated with a wide variety of serious cardiovascular, neurodegenerative or metabolic diseases and at least in some of them, mitochondria are regarded as a key player in the onset or progress of the disease (Javadov et al. 2020). It is also known that many mitochondrial functions decrease with age which might contribute to frailty, sarcopenia, neurodegenerative and metabolic disorders frequently associated with the advanced age (Alexiou et al. 2018, Joseph et al. 2012). Analysis of mitochondrial oxygen consumption and energy production requires fresh samples of the affected tissues obtained by invasive tissue biopsies or use of animal models of the diseases that have frequently only limited translational potential (Leenaars et al. 2019). Over the last decade, a number of studies have documented that the assessment of bioenergetics of peripheral blood cells might reflect mitochondrial dysfunction in metabolically active tissues (Petrus et al. 2019).

Among blood cells equipped with mitochondria, platelets represent an easily accessible and relatively homogenous pool of cells suitable for evaluation of systemic mitochondrial function (Schapira 1993, Tyrrell 
et al. 2014, Tyrrell et al. 2016). Platelets are anucleate cells circulating in the blood and playing an important role in all steps of hemostasis, i.e. vasoconstriction, platelet plug formation, blood coagulation, and tissue repair. However, platelets seem to be involved in many processes beyond the hemostasis including inflammation, immunoregulation and probably also pathogenesis of the diseases having serious impact on overall body homeostasis, e.g. neurodegenerative or cardiovascular disorders (Melchinger et al. 2019, Yu et al. 2020).

The aim of this study was to compare two methods widely used to evaluate mitochondrial respiration in platelets: high resolution respirometry (HRR) based on polarographic detection of oxygen content in analyzed samples (Oxygraph O2k, Oroboros Instruments, Innsbruck, Austria) allowing the complex study of intact or permeabilized cells and metabolic flux analysis (MFA) with the Seahorse bioanalyzer XFp (Agilent, Santa Clara, CA, USA) using small numbers of cells to analyze not only oxygen consumption rate (OCR), but also extracellular acidification rate (ECAR) attributed to glycolytic processes. To verify the effect of aging on platelet mitochondrial function, we included in the study two groups of healthy donors free of any acute disease and selected chronic diseases (diabetes mellitus, chronic heart failure, dysthyroidism, depression, cognitive disorder, cancer, coagulopathy, chronic inflammatory disease) taking 3 selected medications at the maximum and having basic blood parameters within the normal range at the ages 70 to 89 years and 20 to 42 years.

\section{Materials and Methods}

\section{Solutions and chemicals}

The solutions and media used in analyses contained (in $\mathrm{mmol} / \mathrm{l}$ ): MIR05 respiration medium: EGTA $0.5, \mathrm{MgCl}_{2} \cdot 6 \mathrm{H}_{2} \mathrm{O} 3$, potassium lactobionate 60 , taurine $20, \mathrm{KH}_{2} \mathrm{PO}_{4} 10$, HEPES 20 , sucrose 110 , fatty acid free bovine serum albumin $1 \mathrm{~g} / 1, \mathrm{pH} 7.0$ (Pesta and Gnaiger 2012); PBSG medium: $\mathrm{Na}_{2} \mathrm{HPO}_{4} \cdot 7 \mathrm{H}_{2} \mathrm{O}$ 7.5, $\mathrm{NaH}_{2} \mathrm{PO}_{4} \cdot \mathrm{H}_{2} \mathrm{O}$ 2.5, $\mathrm{NaCl} 137, \mathrm{KCl} 2.7$, glucose 10; ATP rate assay medium: Seahorse XF DMEM Medium ${ }^{\circledR}$ supplemented with glucose 5.5 , glutamine 4 , pyruvate 1 , $\mathrm{pH}$ 7.4; procaine solution: procaine hydrochloride 86 and $\mathrm{NaCl}$ 34. If not stated otherwise, chemicals were from Sigma-Aldrich (St. Louis, MO, USA). All chemicals were of analytical grade.

\section{Participants}

Self-sufficient volunteers without cognitive deficit aged 70 to 89 years (OLD; $\mathrm{n}=76$ ) were recruited from the University of the Third Age at the Faculty of Medicine in Pilsen, Charles University, healthy relatives of the staff of the laboratory and friends. Fresh blood from younger blood donors (age 20 to 42 years; YOUNG; $\mathrm{n}=44$ ) was obtained from the Transfusion Department of the Faculty Hospital in Pilsen or from the staff of the laboratory.

Medical history was collected from each participant including the use of medication. All younger participants declared no history of acute or chronic disease and had no medications.

In all participants, basic hematologic parameters were determined by standard laboratory methods (white blood cell count (WBC), red blood cell count (RBC), hemoglobin concentration in the blood $(\mathrm{Hb})$, platelet count (PLT)).

In the group of older adults, basic biochemical and hematological screening was required and all participants underwent cognitive testing using Montreal Cognitive Assessment (MoCA) test to exclude cognitive impairment and Short Physical Performance Battery (SPPB) test to evaluate their physical proficiency. All seniors evaluated themselves as self-sufficient and active persons. Out of 76 volunteers, only 15 could be included in the results of this study: Only seven participants stated no medication and no important medical history (1 of them was then excluded on the basis of biochemical symptoms of acute inflammatory disease), six volunteers took vitamin $\mathrm{D}$, calcium and magnesium for osteoporosis, three atorvastatine at a dose not affecting platelet mitochondrial respiration in humans (Vevera et al. 2016), two were on therapy with proton pump inhibitors, 2 used inhibitors of angiotensin I converting enzyme, one participant was treated with $\beta_{1}$-adrenergic antagonist, one inhaled

$\beta_{2}$-adrenergic agonist and one took a low dose corticosteroid medication for the history of rheumatic disease one year ago. All participants with a history of hyper- or hypothyroidism, diabetes, heart failure, ischemic heart disease, depressive disorder, cognitive disorder, inflammatory disease in recent history, history of thrombosis or embolism or on combined medication with more than three drugs were excluded from the study. Scores of MoCA and SPPB tests in the OLD group verified good cognition and self-sufficiency. As expected, $\mathrm{RBC}$ and $\mathrm{Hb}$ were significantly higher in the YOUNG 
group due to unequal distribution of males and females in both groups. Characteristic of the study participants is shown in Table 1.

The study was approved by the Joint Ethics
Committee of the Faculty of Medicine and Faculty Hospital in Pilsen and it was conducted in accordance with the Declaration of Helsinki. Written consent was obtained from all subjects.

Table 1. Characteristic of the study participants.

\begin{tabular}{|c|c|c|c|}
\hline & YOUNG & OLD & $\mathbf{p}$ \\
\hline$N$ & 44 & 15 & \\
\hline Male/Female & $27 / 17$ & $3 / 12$ & \\
\hline Age & $30.7 \pm 6.2$ & $76 \pm 5$ & $<0.01$ \\
\hline \multirow{8}{*}{ Medications } & \multirow{8}{*}{0} & $6-0$ & \\
\hline & & $6-$ vitamin $\mathrm{D}+$ calcium, magnesium & \\
\hline & & 3 - atorvastatin $10 \mathrm{mg} /$ day & \\
\hline & & 2 - proton pump inhibitors & \\
\hline & & $2-\mathrm{ACE}$ inhibitor & \\
\hline & & $1-\beta_{1}$-adrenergic antagonist & \\
\hline & & $1-\beta_{2}$-adrenergic agonist inhalation & \\
\hline & & 1 - methylprednisolone $2 \mathrm{mg} /$ day & \\
\hline$W B C$ & $6.3 \pm 1.1$ & $7.4 \pm 3.0$ & n.s. \\
\hline$R B C$ & $5.2 \pm 0.2$ & $4.6 \pm 0.4$ & $<0.01$ \\
\hline$H b$ & $151 \pm 9$ & $138 \pm 9$ & $<0.01$ \\
\hline$P L T$ & $224 \pm 41$ & $278 \pm 90$ & n.s. \\
\hline$G L C$ & n.d. & $5.3 \pm 1.0$ & \\
\hline$C R P$ & n.d. & $1.34 \pm 0.57$ & \\
\hline$S P P B$ & n.d. & $11.8 \pm 0.4$ & \\
\hline$M o C A$ & n.d. & $27.2 \pm 2.1$ & \\
\hline
\end{tabular}

WBC $=$ white blood cell count $\left(\times 10^{9} / \mathrm{l}\right), \mathrm{RBC}=$ red blood cell count $\left(\times 10^{12} / \mathrm{l}\right), \mathrm{Hb}=$ hemoglobin concentration in the blood $(\mathrm{g} / \mathrm{l})$; $\mathrm{PLT}=$ platelet count $\left(\times 10^{9} / \mathrm{l}\right)$; GLC=blood glucose concentration $(\mathrm{mmol} / \mathrm{l}) ; \mathrm{CRP}=\mathrm{C}$-reactive protein concentration in the plasma $(\mathrm{mg} / \mathrm{l})$; $\mathrm{SPPB}=$ Short Physical Performance Battery test (maximum score 12); MoCA=Montreal Cognitive Assessment test (maximum score 30); n.d. $=$ not determined; n.s. $=$ not significant.

\section{Isolation of platelets}

Platelets were isolated by differential centrifugation of fresh peripheral venous blood samples (27 $\mathrm{ml}$ per person) with EDTA as anticoagulant (Vacuette ${ }^{\circledR}$ blood collection tubes - Dialab, Prague, Czech Republic). Whole blood was mixed with prostaglandin $E_{1}(62.5 \mathrm{ng} / \mathrm{ml})$ to prevent the activation of platelets during the procedure. Platelet-rich plasma (PRP) was obtained by centrifugation at $300 \times \mathrm{g}$ for $20 \mathrm{~min}$ at $25^{\circ} \mathrm{C}$ with no brake. PRP was transferred to the new tubes, mixed with phosphate buffered saline (PBS) at a $1: 1$ ratio and then centrifuged at $1500 \times \mathrm{g}$ for $10 \mathrm{~min}$ at $25^{\circ} \mathrm{C}$. Supernatants were discarded and the platelet pellets were resuspended in the final media: in MIR05 to measure mitochondrial oxygen consumption in permeabilized platelets and in PBSG to evaluate oxygen consumption in intact platelets. Samples of platelet suspensions were washed for $10 \mathrm{~min}$ in the procaine solution to lyse the red blood cells and counted in Bürker's chamber. Final platelet count was adjusted to $150-350 \times 10^{9} / 1$.

Evaluation of mitochondrial respiration using highresolution respirometry ( $\mathrm{HRR} ; \mathrm{O} 2 \mathrm{k})$

Intact platelets

In the preliminary experiments, the intact platelets were tested in two media: ATP rate assay medium and PBSG. No significant differences were found in platelet oxygen consumption rates between the two media (data not shown) and the intact platelets were then analyzed in PBSG medium at $37^{\circ} \mathrm{C}$. After equilibration of the medium with atmospheric air and subsequent air calibration, the oxygraph chambers were filled with the samples containing platelet suspensions in 
PBSG. The following respiratory states were determined: routine respiration (ROUT) as endogenous basal oxygen consumption dependent on intracellularly available substrates; leak respiration (LEAK) as oxygen consumption determined after administration of ATP-synthase inhibitor oligomycin (Omy; $2 \mu \mathrm{g} / \mathrm{ml}$ ) essential for compensation for the proton leakage; capacity of the electron-transporting system (ETSC) as an uncoupled respiration after titration with the protonophore carbonyl cyanide p-trifluoromethoxyphenylhydrazone (FCCP; $0.5 \mu \mathrm{mol} / 1$ titrations); residual oxygen consumption (ROX) after addition of $0.5 \mu \mathrm{mol} / 1$ rotenone (Rot) and $2.5 \mu \mathrm{g} / \mathrm{ml}$ antimycin A (Ama). A representative graph of an experiment in intact cells is shown in Figure 1A.

\section{Permeabilized platelets (HRR, O2k)}

Permeabilized platelets were evaluated in the MIR05 medium at $37^{\circ} \mathrm{C}$ using the following substrateuncoupler-inhibitor-titration protocol (SUIT; Pesta and Gnaiger 2012). After stabilization of the resting respiration, digitonin (dig; $1 \mu \mathrm{g} / 10^{8}$ platelets) was titrated to permeabilize the platelet membrane and then malate $(0.1 \mathrm{mmol} / \mathrm{l})$ and palmitoyl-L-carnitine (Pcar; $0.04 \mathrm{mmol} / \mathrm{l})$ were added to induce non-phosphorylating leak state when metabolism of fatty acids is activated (LEAK-FAO). Then, active phosphorylating respiration was induced by $5 \mathrm{mmol} / \mathrm{l}$ adenosine diphosphate (D; OXPHOS-FAO) and $10 \mu \mathrm{mol} / 1$ cytochrome c (c) was added to check the integrity of the outer mitochondrial membrane. Glutamate (G; $10 \mathrm{mmol} / \mathrm{l})$ and pyruvate (P; $5 \mathrm{mmol} / \mathrm{l}$ ) were injected as substrates providing electrons to Complex I (OXPHOS-FAO+I), succinate (S; $50 \mathrm{mmol} / \mathrm{l}$ ) to stimulate Complex II (OXPHOSFAO $+\mathrm{I}+\mathrm{II})$, oligomycin $(2 \mu \mathrm{g} / \mathrm{ml})$ to induce the leak state (LEAK-OMY); FCCP titrations $(0.5 \mu \mathrm{mol} / \mathrm{l})$ were used to uncouple phosphorylation and electron transport and to determine the uncoupled state ETSC-FAO+I+II. Complex I inhibitor rotenone $(0.5 \mu \mathrm{mol} / \mathrm{l})$ was then added to induce ETSC-II and antimycin A $(2.5 \mu \mathrm{g} / \mathrm{ml})$ to inhibit Complex III and measure the state ROX. N,N, $\mathrm{N}^{\prime}, \mathrm{N}^{\prime}-$ tetramethyl-p-phenylenediamine (TMPD; $0.5 \mathrm{mmol} / \mathrm{l}$ ) in the presence of ascorbate (Asc; $2 \mathrm{mmol} / \mathrm{l}$ ) was injected to measure the uncoupled capacity of the Complex IV (CIV) that was determined after subtracting the oxygen consumption after administration of sodium azide (Azd; $100 \mathrm{mmol} / \mathrm{l}$ ), the Complex IV inhibitor. All respiratory states were corrected for ROX.

A sample graph of an experiment in permeabilized cells is shown in Figure 1B.
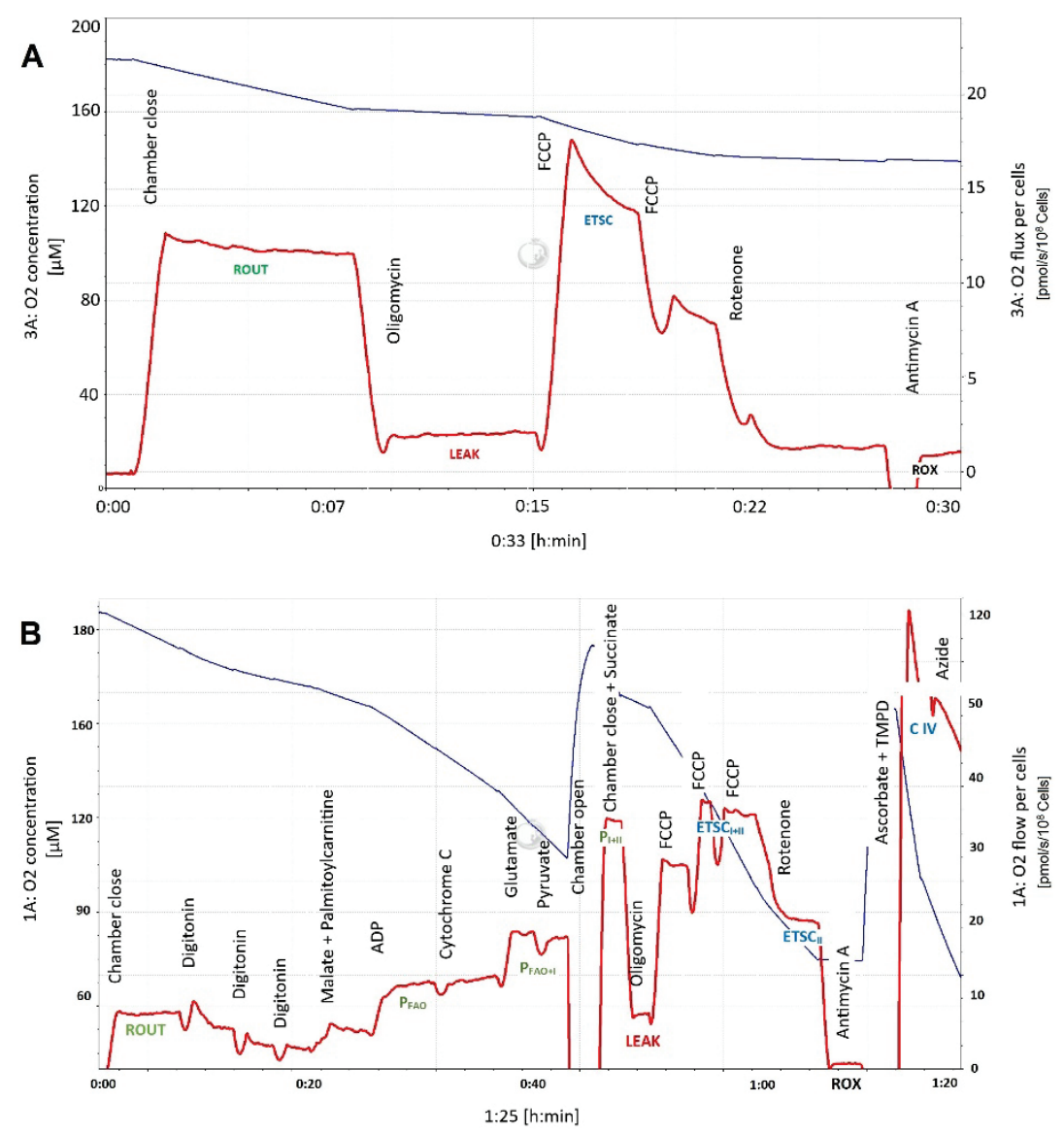

Fig. 1. A representative graph of an experiment in intact (A) and permeabilized platelets (B). Red line oxygen consumption rate, blue line oxygen concentration in the oxygraph chamber. ROUT=routine respiration, $\mathrm{ETSC}=$ capacity of the electrontransporting system, $\mathrm{P}=\mathrm{OXPHOS}$, active phosphorylating respiration with palmitoyl-L-carnitine (FAO), substrates providing electrons to Complex I (I) and succinate (I+II), ETSC II electron-transporting system capacity after inhibition of Complex I by rotenone, CIV=uncoupled respiratory capacity of Complex IV. For the concentrations of substrates, inhibitors and uncoupler utilized and precise definition of respiratory states, see Methods. 
Evaluation of mitochondrial respiration using metabolic flux analysis (MFA; Seahorse XFp)

Simultaneously with determination of oxygen consumption by HRR, platelets $\left(0.8-1.2 \times 10^{7}\right.$ cells per well) were plated on Cell-Tak coated (Corning ${ }^{\circledR}$ CellTak $^{\text {TM }}$ Cell and Tissue Adhesive, Corning, NY, USA) 8 well format XFp plates in the ATP rate assay medium. ATP-rate Real-Time assay (Agilent, Santa Clara, CA, USA) was performed according the manufacturer's instructions. Briefly, five values of basal oxygen consumption rate were measured to determine the state Basal OCR, then oligomycin $(1.0 \mu \mathrm{mol} / \mathrm{l})$ was injected to measure Leak OCR and rotenone $(0.5 \mu \mathrm{mol} / \mathrm{l})+$ antimycin A $(0.5 \mu \mathrm{mol} / \mathrm{l})$ to define ROX OCR. Final values were corrected for ROX. ATP glycolytic and mitochondrial production rates (ATPglyco and ATPmito, respectively) were determined using Seahorse XF RealTime ATP rate Assay Report (Agilent, Santa Clara, CA, USA). A representative graph of an experiment showing OCR and ECAR is displayed in Figure 2A and B.
A

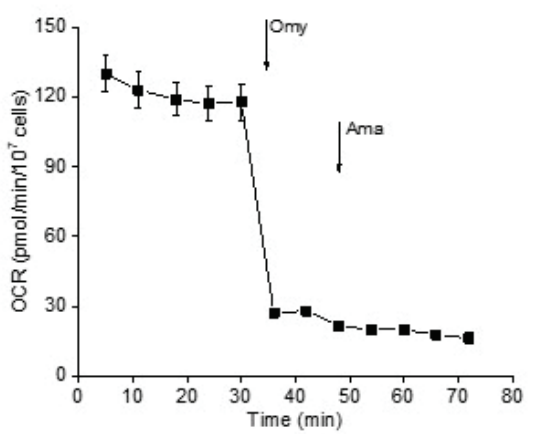

B

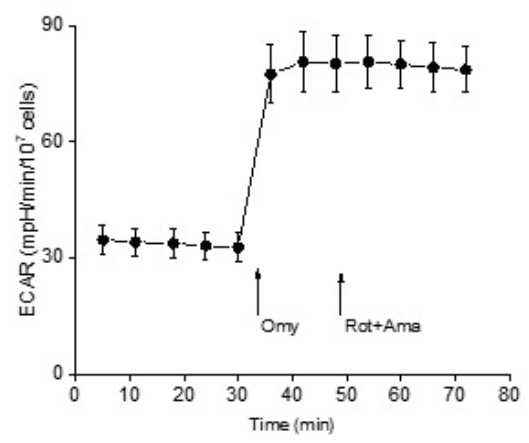

C

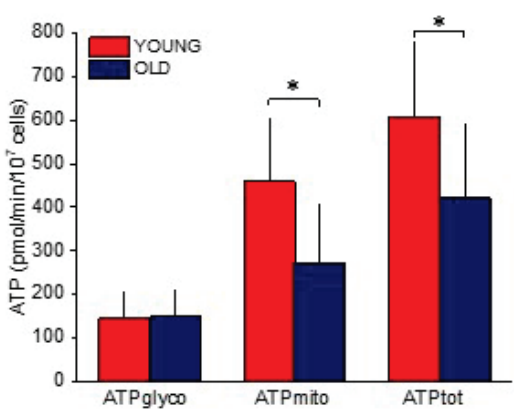

D

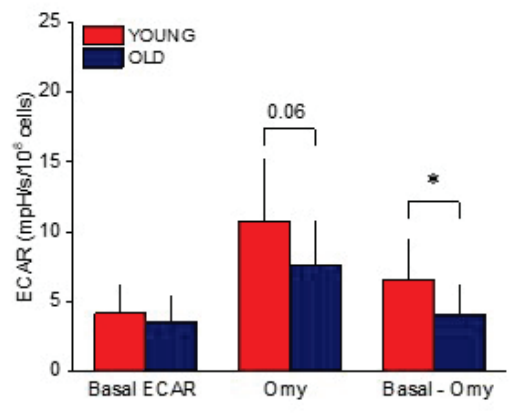

Fig. 2. ATP rate assay. (A) OCR (oxygen consumption rate) in a single experiment. Omy=oligomycin, Ama=antimycin A, Rot=rotenone. (B) ECAR (extracellular acidification rate) in the same single experiment. (C) ATP production rate in the platelets isolated from younger (YOUNG) and older adults (OLD). ATPglyco=glycolytic ATP production, ATPmito $=$ mitochondrial ATP production, ATPtot=total ATP production. (D) ECAR in the platelets isolated from younger (YOUNG) and older adults (OLD). Omy=ECAR after oligomycin, Basal Omy=difference between basal ECAR and ECAR after Omy injection. All bioenergetic measurements were normalized to $10^{7}$ or $10^{8}$ cells. $* \mathrm{p}<0.05$.

\section{Data presentation and statistics}

Results are presented as means $\pm \mathrm{SD}$. After testing for normality of distribution (Shapiro-Wilk test), statistical analyses were performed using the unpaired 2-tailed Student's $t$-test, paired 2-tailed Student's $t$-test or Mann-Whitney U test, where appropriate. To test for significant correlations between mitochondrial parameters acquired by the two methods used, Spearman's rank correlation coefficients $(\rho)$ were calculated. Values of $\mathrm{p}<0.05$ were considered significant. OriginPro 2017 software (OriginLab Corp., Northampton, MA, USA) was used for statistical analyses.

\section{Results}

\section{Intact platelets}

As shown in Table 2, ROUT and ETSC respiratory states determined by HRR were significantly lower in the platelets of the OLD group. Mitochondrial spare respiratory capacity determined by subtracting ROUT respiration from ETSC oxygen consumption (E-R) was also significantly lower in the platelets isolated from older participants (OLD), as was the oxygen consumption related to oxidative ATP production, i.e. the difference between states ROUT and LEAK (R-L). The state corresponding to ROUT respiration determined by MFA (Basal OCR) was also significantly higher in the platelets isolated from younger (YOUNG) compared to older (OLD) participants. In addition, determination of ATP production rate by MFA clearly showed that in the group OLD platelets, mitochondrial and total ATP production was decreased compared to the YOUNG group, but glycolytic ATP synthesis did not significantly differ between the two groups (Fig. 2). 
Extracellular acidification rate (ECAR) roughly reflecting glycolysis did not differ between the two groups, however its increase after oligomycin injection (i.e. after inhibition of ATP-synthase) was significantly lower in the group OLD platelets (Fig. 2).

Table 2. Bioenergetic parameters of intact platelets determined by HRR and MFA.

\begin{tabular}{lccc}
\hline HRR $($ O2k) & YOUNG & OLD & p \\
\hline ROUT & $10.2 \pm 3.0$ & $7.6 \pm 2.5$ & $<0.005$ \\
LEAK & $1.5 \pm 0.6$ & $1.4 \pm 0.7$ & n.s. \\
ETSC & $14.3 \pm 5.7$ & $8.7 \pm 4.4$ & $<0.005$ \\
ROX & $0.8 \pm 0.4$ & $1.0 \pm 0.3$ & n.s. \\
$R-L$ & $8.7 \pm 3.7$ & $5.9 \pm 2.0$ & $<0.001$ \\
E-R & $4.4 \pm 4.2$ & $1.1 \pm 3.4$ & $<0.01$ \\
$R / L$ & $7.4 \pm 1.8$ & $6.3 \pm 2.3$ & n.s. \\
L/E & $0.12 \pm 0.06$ & $0.20 \pm 0.13$ & $<0.05$ \\
MFA (SEAHORSE) & & & $<0.05$ \\
OCR basal & $14.5 \pm 7.9$ & $8.9 \pm 4.0$ & n.s. \\
OCR leak & $2.0 \pm 2.5$ & $1.3 \pm 0.7$ & n.s. \\
OCR rox & $3.5 \pm 2.0$ & $2.2 \pm 0.8$ & $=0.06$ \\
B-L & $11.9 \pm 6.4$ & $7.6 \pm 3.8$ & n.s. \\
B/L & $16.3 \pm 15.3$ & $8.4 \pm 4.8$ & n.s \\
ATP glyco & $146 \pm 59$ & $149 \pm 59$ & $<0.01$ \\
ATP mito & $460 \pm 143$ & $274 \pm 135$ & $<0.05$ \\
ATP tot & $606 \pm 173$ & $422 \pm 169$ & $<0.05$ \\
\hline glyco & $24 \pm 7$ & $37 \pm 13$ & \\
\hline
\end{tabular}

Mitochondrial respiration of intact platelets in younger and older participants of the study (YOUNG and OLD, respectively). Respiratory states are expressed as $\mathrm{pmol} \mathrm{O}_{2} / \mathrm{s} / 10^{8}$ platelets; ATP production rates in $\mathrm{pmol} / \mathrm{min} / 10^{7}$ cells, values are presented as mean $\pm \mathrm{SD}$. ROUT=routine respiration; $E T S C=$ capacity of the electron-transporting system; $R O X=$ residual oxygen consumption; $R-L=$ routine - leak respiration related to oxidative ATP production, net routine respiration; $R / L=$ routine/leak respiration; $L / E=l e a k / E T S C$, coupling control ratio; $\mathrm{E}-\mathrm{R}=$ difference of $\mathrm{ETSC}$ and routine respiration, reserve capacity; OCR - oxygen consumption rate; $\mathrm{B}-\mathrm{L}=$ difference of basal and leak respiration related to oxidative ATP production; $B / L=$ basal/leak respiration; ATP glyco=glycolytic ATP production rate; ATP mito=oxidative ATP production rate; ATP tot=total ATP production rate; $\%$ glyco=glycolytic to total ATP production in $\%$. For precise definition of respiratory states and inhibitors and uncoupler utilized, see Methods. n.s. $=$ not significant.

\section{Permeabilized platelets}

In the YOUNG group platelets, there was a trend to display slightly higher oxygen consumption than in the OLD one in all measured states (Table 3). Significant differences were reached in the states LEAK after malate and palmitoyl carnitine (LEAK-FAO), OXPHOS after succinate (OXPHOS-FAO+I+II), ETSC after FCCP (ETSC-FAO+I+II) and rotenone (ETSC-II); the most prominent decline in the group OLD platelets was observed after TMPD (CIV; Table 3 ). In the platelets isolated from the older adults (OLD), phosphorylating states were decreased by 20 to $24 \%$, uncoupled states by 25 to $44 \%$.

\section{Seahorse and $O 2 k$}

Resting oxygen consumption rates of the intact platelets determined by both methods (i.e. ROUT in HRR and Basal OCR in MFA) significantly correlated $(\mathrm{R}=0.64 ; \mathrm{p}<0.001)$; however expressed per the same unit (i.e. pmol $\mathrm{O}_{2} / 10^{8}$ cells/s), platelets measured by MFA displayed significantly higher OCRs than those measured by HRR $(p<0.01)$. Additional significant correlations were found between similar parameters measured by HRR (O2k) or MFA (Seahorse) in intact cells: $\rho$ was 0.55 $(\mathrm{p}<0.001)$ for R-L $($ HRR) and Basal OCR-Leak OCR (MFA) and $0.43 \quad(\mathrm{p}<0.05)$ for R-L $($ HRR $)$ and mitochondrial ATP production (MFA). Basal OCR also significantly correlated with ETSC in the intact cells $(\rho=0.49 ; p<0.05)$. 
Table 3. Respiratory parameters of permeabilized platelets determined by HRR.

\begin{tabular}{lccccc}
\hline STATE & SUIT & YOUNG & OLD & p & O/Y \% \\
\hline ROUT & & $7.03 \pm 2.4$ & $6.4 \pm 1.9$ & n.s. & 91 \\
& $+\mathrm{dig}$ & $2.9 \pm 1.3$ & $1.7 \pm 0.9$ & & \\
LEAK-FAO & $+\mathrm{M}+$ Pcar & $5.0 \pm 1.6$ & $3.5 \pm 1.6$ & $<0.01$ & 70 \\
OXPHOS-FAO & $+\mathrm{D}$ & $9.4 \pm 5.2$ & $7.1 \pm 4.0$ & n.s. & 76 \\
OXPHOS-FAOc & $+\mathrm{c}$ & $9.2 \pm 5.5$ & $7.6 \pm 4.2$ & n.s. & 83 \\
OXPHOS-FAO+I & $+\mathrm{G}$ & $13.1 \pm 8.3$ & $10.6 \pm 5.7$ & n.s. & 81 \\
OXPHOS-FAO+IP & $+\mathrm{P}$ & $12.8 \pm 8.0$ & $10.1 \pm 5.4$ & n.s. & 79 \\
OXPHOS-FAO+I+II & $+\mathrm{S}$ & $24.8 \pm 10.5$ & $18.9 \pm 7.1$ & $<0.05$ & 76 \\
LEAK-OMY & + Omy & $5.6 \pm 1.6$ & $5.1 \pm 1.3$ & n.s. & 91 \\
ETSC-FAO+I+II & + FCCP & $27.4 \pm 13.2$ & $19.6 \pm 8.8$ & $<0.05$ & 72 \\
ETSC-II & + Rot & $13.8 \pm 6.6$ & $8.9 \pm 4.9$ & $<0.05$ & 64 \\
CIV & + TMPD/Asc + & $76.5 \pm 23.5$ & $42.6 \pm 12.9$ & $<0.0001$ & 56 \\
\hline
\end{tabular}

Mitochondrial respiration of permeabilized platelets in YOUNG $(Y)$ and OLD (O) participants of the study. Respiratory states are expressed as pmol $\mathrm{O}_{2} / \mathrm{s} / 10^{8}$ platelets; values are presented as mean $\pm \mathrm{SD}$. n.s. $=$ not significant. ROUT=routine respiration; LEAK-FAO=leak respiration after addition of palmitoyl-L-carnitine; OXPHOS=active phosphorylating respiration with palmitoyl-Lcarnitine, substrates providing electrons to Complex I (I) and succinate (I+II); LEAK-OMY=leak respiration after addition of oligomycin; ETSC-II=electron-transporting system capacity after inhibition of Complex I by rotenone; CIV=uncoupled respiratory capacity of

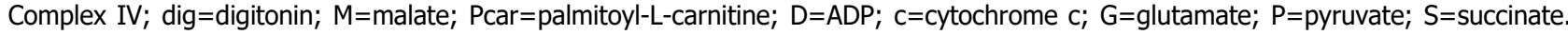
For the concentrations of substrates, inhibitors and uncoupler utilized, see Methods.

\section{Discussion}

Over the last decade, a number of studies have explored the potential use of mitochondrial analysis of peripheral blood cells to judge on overall mitochondrial condition of organs that are not accessible in vivo (Petrus et al. 2019). Evaluation of bioenergetic function of circulating cells might help in search for easily detectable biomarkers in early diagnosis of neurodegenerative or inflammatory diseases (Fišar et al. 2016, Sjövall et al. 2013) and/or monitoring the effects of therapeutic interventions, like physical training (Gatterer et al. 2018) or diet (Bajracharya et al. 2019). However, the results of many studies are conflicting showing either significant correlations between bioenergetic parameters of the blood cells and various organs (Tyrrell et al. 2016, Braganza et al. 2019) or declaring no relationship between mitochondrial respiratory variables measured in circulating cells and skeletal muscle (Hedges et al. 2019, Rose et al. 2019).

In the present study, we assessed mitochondrial respiration of platelets isolated from younger and older adults using two methods, HRR and MFA to cover more aspects of platelet mitochondrial physiology and ATP-production processes. Using HRR, we measured oxygen consumption in permeabilized platelets enabling determination of phosphorylating and electrontransporting capacities of the respiratory complexes in a sequential manner; evaluation of mitochondrial respiration in intact platelets was used to estimate bioenergetic profile of platelets resembling the in vivo conditions (Pesta and Gnaiger 2012). MFA performed in parallel on the same platelets allowed us to compare basal oxygen consumption, leak respiration and residual oxygen consumption to corresponding respiratory states acquired by HRR and to evaluate ATP production rate using a reliable method (Mookerjee et al. 2017). We have decided to use isolated platelets for two reasons: 1. The isolation procedure of platelets is faster and simpler than isolation of peripheral blood mononuclear cell (PBMC) or even individual subtypes of white blood cells; 2. Platelets, although anuclear, represent relatively homogenous source of mitochondria unlike PBMC, consisting of monocytes and lymphocytes that have different bioenergetic profiles (Chacko et al. 2013).

It is generally accepted that aging is associated with a decline of many mitochondrial functions including oxygen consumption rate and ATP production (Boengler et al. 2017). Ample evidence of compromised mitochondrial function exists for aged skeletal muscle: multiple studies documented declining ATP production rate, increased $\mathrm{H}^{+}$permeability of the inner membrane, 
reduced mitochondrial electron-transporting capacity or decreased activities of complexes I and IV (Boffoli et al. 1994, Cooper et al. 1992, Navarro and Boveris 2007, Porter et al. 2015). Similar findings were reported also in the heart, brain, liver or kidney (Choksi et al. 2011, Ojaimi et al. 1999). The reports of age-related decline in platelet mitochondrial respiration or ATP generation are far less numerous. In the study of Sjövall et al. (2013) performed on intact and permeabilized platelets of adults aged 19-65 years and children (1 month-12 years), mitochondrial oxygen consumption was determined using HRR. ROUT respiration tended to increase with age, whereas ETSC-II oxygen consumption displayed an opposite trend. These changes were attributed to the differences between the pediatric and adult cohorts included in the research. In our study, HRR variables in both intact and permeabilized platelets had comparable absolute values; however, more statistically significant differences we found: platelets isolated from older adults displayed significantly lower uncoupled respirations, i.e. ETSC-ETF+I+II, ETSC-II, CIV (permeabilized cells) and ETSC (intact platelets) than those acquired from younger participants. In addition, besides lower uncoupled respiratory states, intact platelets isolated from older participants showed also lower routine respiration, ATP-linked oxygen consumption (R-L), and reserve capacity (E-R) than those isolated from younger donors. This difference could be related to the age of our older volunteers (at least 70 years) compared to the Swedish cohort (65 years at the maximum). In another study performed on platelets isolated from young (age 18-35 years) and older (86-93 years) adults using MFA (Braganza et al. 2019), basal OCR and ATP-linked OCR were lower in older population, which is concordant with our study.

The relative contribution of the energygenerating processes in the platelets (i.e. glycolysis and oxidative phosphorylation) is still a matter of debate. According to the published data, mitochondrial ATP production accounts for 35 to $85 \%$ of the energy production and glycolysis for remaining 15 to $65 \%$ (Kilkson et al. 1984, Ravi et al. 2015, Akkerman and Holmsen 1981). The results of our study show that mitochondrial ATP production represented $75 \%$ of the total ATP synthesis rate in young adults and that it was significantly decreased in the platelets of aged persons. In contrast, ATP generation linked to glycolysis was well preserved even in the advanced age. Positive significant correlation between the R-L parameter measured in the intact cells by HRR and mitochondrial ATP production determined by MFA confirms that this variable can be taken as a rough estimation of ATP production in mitochondria also when HRR is used and corroborates good compatibility of both methods. Studies dealing with the impact of aging on platelet ATP production are rare. Shi et al. (2008) reported no difference in ATP content in platelets between young and aged healthy subjects, others judged on age-related decreasing ATP production in the platelets from oxygen consumption rates (Braganza et al. 2019).

\section{Study limitations}

Although both methods used for evaluation of mitochondrial respiration and extramitochondrial ATP production in this study provided comparable results, it should be noted that respiratory rates measured by MFA were significantly higher than corresponding mitochondrial parameters acquired by HRR. We believe that it could be related to different status of platelets in O2k oxygraph chambers where the platelets are continuously stirred and during the assay, it is possible to check their potential aggregation, and Seahorse analyzer where the platelets have to be stuck on the bottom of the well and their at least partial mechanical activation cannot be excluded (Qiu et al. 2014).

Another issue is the recruitment of the control volunteers at the advanced age (Malay and Chung 2012, Mody et al. 2008). As noted above, in this study, only 7 participants (of 76) declared no important medical history and did not take any medication. The extent to which such a selection could be regarded as a representative control group if platelet mitochondrial parameters are evaluated as a potential marker of various diseases is questionable. Altered platelet mitochondrial consumption has been found in a number of diseases that are frequently diagnosed simultaneously in older people (Petrus et al. 2019, Wang et al. 2017). Nevertheless, in this study, we aimed to assess the impact of aging per se on platelet bioenergetics.

The potential impact of gender could not be addressed in this study due to unequal distribution of males and females in the OLD group. However, no correlation between respiratory parameters and gender were seen neither in intact nor permeabilized platelets in the study of Sjövall et al. (2013). 


\section{Conclusions}

We have verified compromised mitochondrial respiration and oxidative ATP production in the platelets of older persons. In addition, our study documents good compatibility of the two most widely used methods for determining the global performance of the electrontransporting system, i.e. HRR and MFA.

\section{Conflict of Interest}

There is no conflict of interest.

\section{Acknowledgements}

This research was funded by the project of the Grant Agency of the Charles University No. 966120, the Charles University Research Fund (project no. Q39), project No. CZ.02.1.01/0.0/0.0/16_019/0000787 Fighting Infectious Diseases awarded by the MEYS CR, financed from EFRR, project No. CZ.02.1.01/0.0/0.0/17_048/ 0007267, "Research and Development of Intelligent Components of Advanced Technologies for the Pilsen Metropolitan Area (InteCom)", awarded by the MEYS CR, financed from ERDF CZ, and the Specific Student Research Project Nr. 260538/2020 of the Charles University.

\section{References}

AKKERMAN JW, HOLMSEN H: Interrelationships among platelet responses: studies on the burst in proton liberation, lactate production, and oxygen uptake during platelet aggregation and Ca2+ secretion. Blood 57: 956-966, 1981. https://doi.org/10.1182/blood.V57.5.956.bloodjournal575956

ALEXIOU A, NIZAMI B, KHAN FI, SOURSOU G, VAIRAKTARAKIS C, CHATZICHRONIS S, TSIAMIS V, MANZTAVINOS V, YARLA NS, MD ASHRAF G: Mitochondrial dynamics and proteins related to neurodegenerative diseases. Curr Protein Pept Sci 19: 850-857, 2018. https://doi.org/10.2174/1389203718666170810150151

BAJRACHARYA R, YOUNGSON NA, BALLARD JWO: Dietary macronutrient management to treat mitochondrial dysfunction in Parkinson's disease. Int J Mol Sci 20: 1850, 2019. https://doi.org/10.3390/ijms20081850

BOENGLER K, KOSIOL M, MAYR M, SCHULZ R, ROHRBACH S: Mitochondria and ageing: Role in heart, skeletal muscle and adipose tissue. J Cachexia Sarcopenia Muscle 8: 349-369, 2017. https://doi.org/10.1002/jcsm.12178

BOFFOLI D, SCACCO SC, VERGARI R, SOLARINO G, SANTACROCE G, PAPA S: Decline with age of the respiratory chain activity in human skeletal muscle. Biochim Biophys Acta 1226: 73-82, 1994. https://doi.org/10.1016/09254439(94)90061-2

BRAGANZA A, COREY CG, SANTANASTO AJ, DISTEFANO G, COEN PM, GLYNN NW, NOURAIE SM, GOODPASTER BH, NEWMAN AB, SHIVA S: Platelet bioenergetics correlate with muscle energetics and are altered in older adults. JCI Insight 5: e128248, 2019. https://doi.org/10.1172/jci.insight.128248

CHACKO BK, KRAMER PA, RAVI S, JOHNSON MS, HARDY RW, BALLINGER SW, DARLEY-USMAR VM: Methods for defining distinct bioenergetic profiles in platelets, lymphocytes, monocytes, and neutrophils, and the oxidative burst from human blood. Lab Invest 93: 690-700, 2013. https://doi.org/10.1038/labinvest.2013.53

CHOKSI KB, NUSS JE, DEFORD JH, PAPACONSTANTINOU J: Mitochondrial electron transport chain functions in long-lived Ames dwarf mice. Aging 3: 754-767, 2011. https://doi.org/10.18632/aging.100357

COOPER JM, MANN VM, SCHAPIRA AH: Analyses of mitochondrial respiratory chain function and mitochondrial DNA deletion in human skeletal muscle: effect of ageing. J Neurol Sci 113: 91-98, 1992. https://doi.org/10.1016/0022-510X(92)90270-U

FIŠAR Z, HROUDOVÁ J, HANSÍKOVÁ H, SPÁČILOVÁ J, LELKOVÁ P, WENCHICH L, JIRÁK R, ZVĚŘOVÁ M, ZEMAN J, MARTÁSEK P, RABOCH J: Mitochondrial respiration in the platelets of patients with Alzheimer's disease. Curr Alzheimer Res 2016. https://doi.org/10.2174/1567205013666160314150856

GATTERER H, MENZ V, SALAZAR-MARTINEZ E, SUMBALOVA Z, GARCIA-SOUZA LF, VELIKA B, GNAIGER E, BURTSCHER M: Exercise performance, muscle oxygen extraction and blood cell mitochondrial respiration after repeated-sprint and sprint interval training in hypoxia: A pilot study. J Sports Sci Med 17: $339-347,2018$. 
HEDGES CP, WOODHEAD JST, WANG HW, MITCHELL CJ, CAMERON-SMITH D, HICKEY AJR, MERRY TL: Peripheral blood mononuclear cells do not reflect skeletal muscle mitochondrial function or adaptation to highintensity interval training in healthy young men. J Appl Physiol 126: 454-461, 2019. https://doi.org/10.1152/japplphysiol.00777.2018

JAVADOV S, KOZLOV AV, CAMARA AKS: Mitochondria in health and diseases. Cells 9: 1177, 2020. https://doi.org/10.3390/cells9051177

JOSEPH AM, ADHIHETTY PJ, BUFORD TW, WOHLGEMUTH SE, LEES HA, NGUYEN LM, ARANDA JM, SANDESARA BD, PAHOR M, MANINI TM, MARZETTI E, LEEUWENBURGH C: The impact of aging on mitochondrial function and biogenesis pathways in skeletal muscle of sedentary high- and low-functioning elderly individuals. Aging Cell 11: 801-809, 2012. https://doi.org/10.1111/j.1474-9726.2012.00844.x

KILKSON H, HOLME S, MURPHY S: Platelet metabolism during storage of platelet concentrates at 22 degrees C. Blood 64: 406-414, 1984. https://doi.org/10.1182/blood.V64.2.406.bloodjournal642406

LEENAARS CHC, KOUWENAAR C, STAFLEU FR, BLEICH A, RITSKES-HOITINGA M, DE VRIES RBM, MEIJBOOM FLB: Animal to human translation: a systematic scoping review of reported concordance rates. J Transl Med 17: 223, 2019. https://doi.org/10.1186/s12967-019-1976-2

MALAY S, CHUNG KC: The choice of controls for providing validity and evidence in clinical research. Plast Reconstr Surg 130: 959-965, 2012. https://doi.org/10.1097/PRS.0b013e318262f4c8

MELCHINGER H, JAIN K, TYAGI T, HWA J: Role of platelet mitochondria: Life in a nucleus-free zone. Front Cardiovasc Med 6: 153, 2019. https://doi.org/10.3389/fcvm.2019.00153

MODY L, MILLER DK, MCGLOIN JM, FREEMAN M, MARCANTONIO ER, MAGAZINER J, STUDENSKI S: Recruitment and retention of older adults in aging research. J Am Geriatr Soc 56: 2340-2348, 2008. https://doi.org/10.1111/j.1532-5415.2008.02015.x

MOOKERJEE SA, GERENCSER AA, NICHOLLS DG, BRAND MD: Quantifying intracellular rates of glycolytic and oxidative ATP production and consumption using extracellular flux measurements. J Biol Chem 292: 7189-7207, 2017. https://doi.org/10.1074/jbc.M116.774471

NAVARRO A, BOVERIS A: The mitochondrial energy transduction system and the aging process. Am J Physiol Cell Physiol 292: C670-C686, 2007. https://doi.org/10.1152/ajpcell.00213.2006

OJAIMI J, MASTERS CL, OPESKIN K, MCKELVIE P, BYRNE E: Mitochondrial respiratory chain activity in the human brain as a function of age. Mech Ageing Dev 111: 39-47, 1999. https://doi.org/10.1016/S0047-6374(99)00071-8

PESTA D, GNAIGER E: High-resolution respirometry: OXPHOS protocols for human cells and permeabilized fibers from small biopsies of human muscle. Methods Mol Biol 810: 25-58, 2012. https://doi.org/10.1007/978-1-61779-382-0_3

PETRUS AT, LIGHEZAN DL, DANILA MD, DUICU OM, STURZA A, MUNTEAN DM, IONITA I: Assessment of platelet respiration as emerging biomarker of disease. Physiol Res 68: 347-363, 2019. https://doi.org/10.33549/physiolres.934032

PORTER C, HURREN NM, COTTER MV, BHATTARAI N, REIDY PT, DILLON EL, DURHAM WJ, TUVDENDORJ D, SHEFFIELD-MOORE M, VOLPI E, SIDOSSIS LS, RASMUSSEN BB, BØRSHEIM E: Mitochondrial respiratory capacity and coupling control decline with age in human skeletal muscle. Am J Physiol Endocrinol Metab 309: E224-E232, 2015. https://doi.org/10.1152/ajpendo.00125.2015

QIU Y, BROWN AC, MYERS DR, SAKURAI Y, MANNINO RG, TRAN R, AHN B, HARDY ET, KEE MF, KUMAR S, BAO G, BARKER TH, LAM WA: Platelet mechanosensing of substrate stiffness during clot formation mediates adhesion, spreading, and activation. Proc Natl Acad Sci U S A 111: 14430-14435, 2014. https://doi.org/10.1073/pnas.1322917111

RAVI S, CHACKO B, SAWADA H, KRAMER PA, JOHNSON MS, BENAVIDES GA, O'DONNELL V, MARQUES MB, DARLEY-USMAR VM: Metabolic plasticity in resting and thrombin activated platelets. PLoS One 10: e0123597, 2015. https://doi.org/10.1371/journal.pone.0123597

ROSE S, CARVALHO E, DIAZ EC, COTTER M, BENNURI SC, AZHAR G, FRYE RE, ADAMS SH, BØRSHEIM E: A comparative study of mitochondrial respiration in circulating blood cells and skeletal muscle fibers in women. Am J Physiol Endocrinol Metab 317: E503-E512, 2019. https://doi.org/10.1152/ajpendo.00084.2019

SCHAPIRA AH: Mitochondrial complex I deficiency in Parkinson's disease. Adv Neurol 60: 288-291, 1993. 
SHI C, GUO K, YEW DT, YAO Z, FORSTER EL, WANG H, XU J: Effects of ageing and Alzheimer's disease on mitochondrial function of human platelets. Exp Gerontol 43: 589-594, 2008. https://doi.org/10.1016/j.exger.2008.02.004

SJÖVALL F, EHINGER JK, MARELSSON SE, MOROTA S, FROSTNER EA, UCHINO H, LUNDGREN J, ARNBJÖRNSSON E, HANSSON MJ, FELLMAN V, ELMÉR E: Mitochondrial respiration in human viable platelets - methodology and influence of gender, age and storage. Mitochondrion 13: 7-14, 2013. https://doi.org/10.1016/j.mito.2012.11.001

TYRRELL DJ, BHARADWAJ MS, JORGENSEN MJ, REGISTER TC, MOLINA AJ: Blood cell respirometry is associated with skeletal and cardiac muscle bioenergetics: Implications for a minimally invasive biomarker of mitochondrial health. Redox Biol 10: 65-77, 2016. https://doi.org/10.1016/j.redox.2016.09.009

TYRRELL DJ, BHARADWAJ MS, VAN HORN CG, KRITCHEVSKY SB, NICKLAS BJ, MOLINA AJ: Respirometric profiling of muscle mitochondria and blood cells are associated with differences in gait speed among communitydwelling older adults. J Gerontol A Biol Sci Med Sci 70: 1394-1399, 2014. https://doi.org/10.1093/gerona/glu096

VEVERA J, FIŠAR Z, NEKOVÁŘOVÁ T, VRABLÍK M, ZLATOHLÁVEK L, HROUDOVÁ J, SINGH N, RABOCH J, VALEŠ K: Statin-induced changes in mitochondrial respiration in blood platelets in rats and human with dyslipidemia. Physiol Res 65: 777-788, 2016. https://doi.org/10.33549/physiolres.933264

WANG L, WU Q, FAN Z, XIE R, WANG Z, LU Y: Platelet mitochondrial dysfunction and the correlation with human diseases. Biochem Soc Trans 45: 1213, 2017. https://doi.org/10.1042/BST20170291

YU H, HU W, SONG X, ZHAO Y: Immune modulation of platelet-derived mitochondria on memory CD4(+) T cells in humans. Int J Mol Sci 21: 6295, 2020. https://doi.org/10.3390/ijms21176295 